\title{
Atomic Force and Confocal Microscopy Analysis of Mechanically Stressed Endothelial Cells Involved in Wound Healing
}

\author{
N. Levi, M.J. Morykwas, ${ }^{* *}$ and D.L. Carroll* \\ *Center for Nanotechnology and Molecular Materials, Department of Physics, Wake \\ Forest University, Winston-Salem, NC 27105 \\ **Department of Plastic and Reconstructive Surgery, Wake Forest University School of \\ Medicine, Winston-Salem, NC 27157
}

Large or chronic wounds present a challenge to both physicians and patients but application of a vacuum assisted closure device (V.A.C.) can offer significant progression in the healing of such wounds [1]. The V.A.C. applies subatmospheric pressure, continuously or cyclically, to the wound site, which reduces edema, and promotes granulation tissue formation [2]. In addition to these benefits, a linear stress is applied to cells in the tissue adjacent to the wound, which has positive effect on the healing rate. Although the clinical results of the V.A.C. method are impressive, there remain significant gaps in understanding how linear stresses affect wound healing at the cellular level, especially when cyclically applied subatmospheric pressure treatments are used.

Previous work has been done regarding the examination of cells and subcellular components using AFM, confocal microscopy, and histochemical analysis [3-6]. However, dynamic experiments using AFM have only involved shear stress using fluid flow in a wet cell [7]. In situ, uniaxial cell stretching experiments using light microscopy have been done [8], but we are using fluorescent confocal microscopy of stretched live cells to obtain better resolution of cytoskeletal filaments to compare with our AFM results. To our knowledge, no group has attempted a dynamical examination of applied one-dimensional mechanical stress to live cells using AFM or confocal microscopy. Atomic force microscopy provides an excellent technique to examine changes in the cytoskeleton of stretched cells, and this can be done repeatedly on live cells adhered to flexible silicone substrates. Fluorescent staining of the cytoskeletal filaments allows a secondary technique to examine dynamic morphological changes using confocal microscopy. Comparison of confocal and AFM results allows for the most definitive conclusions about our results to be made.

Although we are exploring how cytoskeletal filaments will deform as the cell undergoes a linear stretch, we are looking at other subcellular structures, such as the nucleus, for deformation as well. AFM allows us to determine changes in elasticity in various parts of the cell and this information may be compared to histochemical analysis since previous literature has shown that changes in elasticity in specific cell types is related to changes in the production of extracellular matrix proteins [9].

In addition to the novel microscopy techniques, a finite element model is being developed to gain insight to the results. The model calculates continuous and cyclical linear stresses applied as well as the strain. Results of the model can be compared to AFM data of changes in cell elasticity and strain components in specific regions of the cell. 


\section{References:}

[1] Morykwas, M.J., et al., Ann. Plastic Surgery, 28 (1997) 553.

[2] Argenta, L.C., and Morykwas, M.J., Ann. Plastic Surgery, 38 (1997) 563.

[3] Malek, A.M., and Izumo, S., J. Cell Sci., 109 (1996) 713.

[4] Yoshigi, M., et. al., Cytometry, 55A (2003) 109.

[5] Rotsc,h C. and Radmacher, M., Biophys. J., 78 (2000) 520.

[6] Haga, H., et. al., Ultramicro., 82 (2000) 253.

[7] Sato, M., et. al., J. Biomech., 33 (2000) 127.

[8] Naruse, K., et. al., Amer. Physiol. Soc., 274 (1998) 1532.

[9] Yang, G., Et. al., J. Biomech., 37 (2004) 1543.

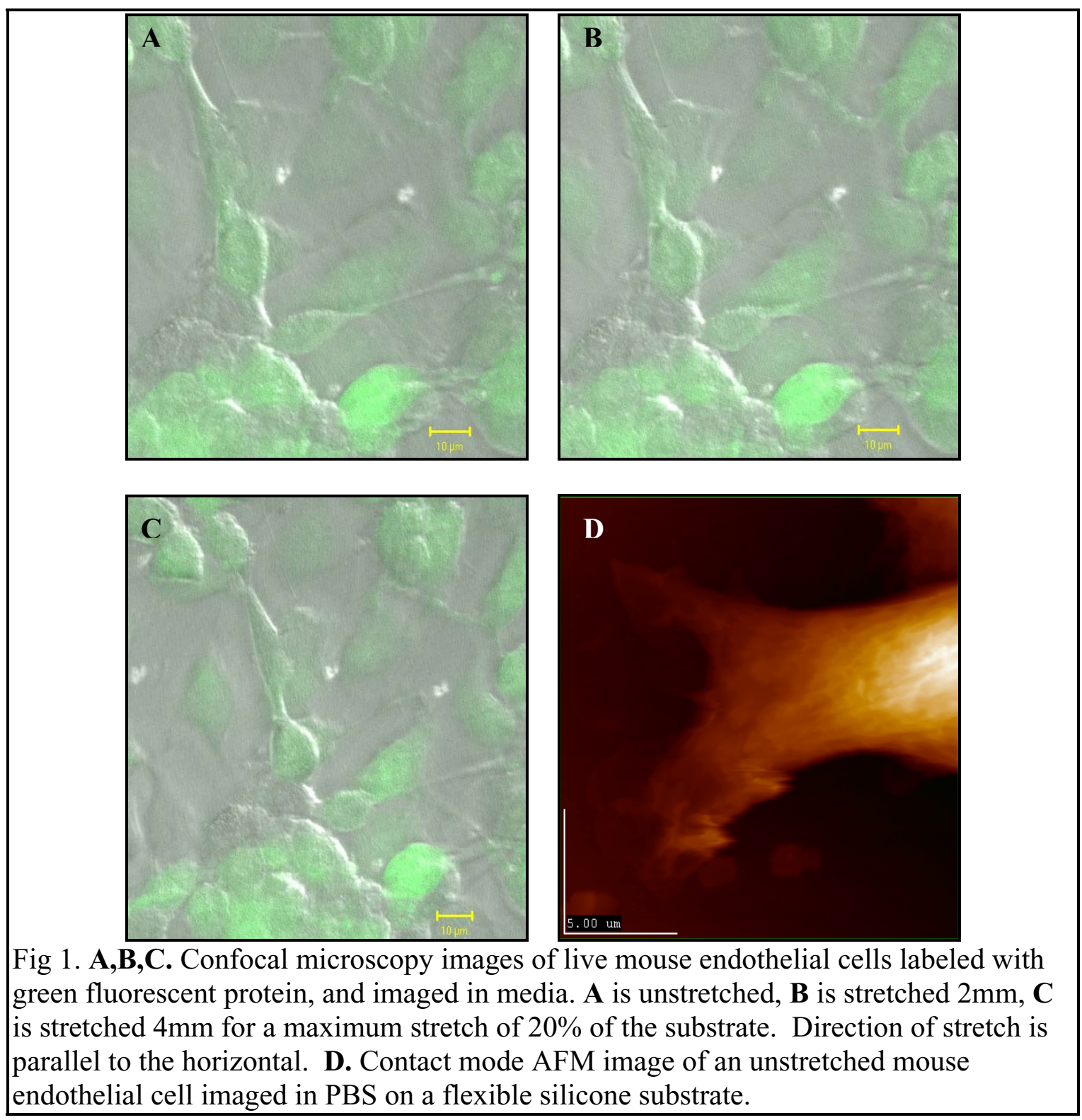

